\title{
Integrating Sustainability in Decision-Making Processes: A Modelling Strategy
}

\author{
Jordi Cabot, Steve Easterbrook, Jennifer Horkoff, Jose-Norberto Mazón, Lysanne Lessard and Sotirios Liaskos \\ Computer Science Department \\ University of Toronto \\ Toronto, Canada \\ jcabot,sme,jenhork,jnmazon,liaskos@cs.toronto.edu, lysanne.lessard@utoronto.ca
}

\begin{abstract}
One of the most difficult problems that humanity currently faces is the sustainable development of our society, i.e. how to meet the needs of the present without compromising the ability of future generations to meet their own needs. Solving this challenge requires a multidisciplinary approach where researchers with different backgrounds combine their efforts. As software engineers, we can contribute to these efforts by offering existing software requirements modeling and analysis techniques as a means to integrate sustainability requirements in decision-making processes. In this paper, we use a popular language for modeling early requirements as a way to visualize the impact of alternative options on sustainability goals and to analyze the conflicts between sustainability and other problem-specific objectives. We apply this idea to the decision-making activities pertaining to the organization of the ICSE'09 conference itself. We find that the modeling activity significantly facilitates exploration, understanding and comparison of large numbers of possible sustainability measures and allows for better informed and justified decisions.
\end{abstract}

\section{Introduction}

Sustainability is the capacity to maintain a certain process or state indefinitely. In particular, we will focus on natural sustainability which is defined as the exploitation of an (eco)system that does not degrade or adversely change the system beyond what is acceptable [1]. Unfortunately, there are growing concerns about the accelerating deterioration of the environment and natural resources. The UN General Assembly recognized that these environmental problems were global in nature and determined that it was in the common interest of all nations to establish policies for a more sustainable development (Brundtland Report [2]). Stricter governmental regulations are forcing all organizations to review their business practices and make them more sustainable. Therefore, the achievement of sustainable practices is a major challenge facing organizations and society as a whole.

In this paper, we suggest that software engineers can contribute to this challenge in two significant ways:
1) By adapting existing $\mathrm{SE}$ techniques for modelling and reasoning about sustainability aspects within an organization or business activity.

2) By integrating sustainability concerns in the development of software systems, whose function has a clear impact on their users' ecofootprint ${ }^{1}$. Moreover, systems should be build in a more flexible way that facilitates "greener" options, i.e. allowing the users to make better choices if they desire, even if that alternative is not the one the system would automatically select (e.g. due to its cost).

In both scenarios, sustainability can be seen as an additional requirement that the business/system must satisfy. In this sense, the main contribution of this paper is to explore a preliminary method for modelling and integrating sustainability issues in the analysis of business alternatives (in general) and software designs (in particular) through the use of $i^{*}$, a popular language for representing and reasoning about stakeholder goals in the early requirements analysis phase [4]. Our approach is based on explicitly representing the sustainability effect of each business or design alternative, in order to allow stakeholders to understand the tradeoffs between sustainability and other business goals and making optimal or otherwise informed decisions.

Our approach complements existing sustainability-specific decision-making frameworks which focus on the development of indicators and mathematical models to optimize the sustainability of a certain activity [5]-[7]. The problem with these approaches is that they optimize the sustainability of the activity without taking into account other business requirements. Instead, we treat sustainability as a tradeoff between the different business goals (usually hindered when favouring a more sustainable approach), which, in our opinion, is more realistic. In addition, our approach aims at engaging communities of stakeholders in the development of the sustainability model, triggering exploratory discussions, improving the validity of the resulting model and fostering common understanding regarding sustainability matters.

1. Ecological footprint assessments allow researchers to analyze whether the amount of biotically productive area available to an economy is equal to or greater than that required to supply all consumed resources and to absorb all generated wastes. This precondition is necessary (although not sufficient) for sustainability [3]. 


\section{Our method for sustainability modeling}

Dealing with sustainability is a complex multi-stakeholder problem that must be addressed at different organizational levels. Improving the sustainability of an activity usually implies hindering other areas of the business/system. Therefore, the trade-offs of each alternative must be carefully evaluated and reconciled by the involved stakeholders before reaching a common agreement. The responsibilities of each stakeholder must also be clarified in order to ensure they comply with sustainability efforts.

These kinds of complex multi-stakeholder problems have been successfully addressed in the requirements engineering community by means of goal-oriented techniques (GORE) [8]. GORE emphasizes the analysis of stakeholders goals and how they might be impacted by different design alternatives. This analysis facilitates communication between stakeholders and guides the consideration and comparison of alternative solutions based on qualitative information (e.g. expert judgement) rather than actual data. A popular GORE modeling language is $i^{*}$ [4]. The $i^{*}$ framework can be used to represent stakeholders (actors in the $i^{*}$ notation), their inter-dependencies, their goals and the (positive or negative) effects of these goals on each other. Goals can be hardgoals (functional requirements) or soft-goals (non-functional requirements). See [4] for more information on the $i^{*}$ syntax.

We propose to use the $i^{*}$ framework to integrate sustainability concerns in either the selection of a design alternative during the development of software system or in the decision-making process during the definition of a business activity. More specifically, we propose to:

1) Define sustainability as a new goal that the business/system must accomplish.

2) Specify the contribution of each possible alternative to this goal so that we can take into account sustainability factors when selecting the best alternative.

We define sustainability as a softgoal (i.e. non-functional requirement) as we cannot have fully attained sustainable solutions, but just options that are more sustainable than others. Furthermore, since sustainability is a complex and multi-faceted notion, this single softgoal is in fact supported by a taxonomy of softgoals that contribute to sustainability in different ways (see an excerpt in Fig. 1). This taxonomy is domain-independent ${ }^{2}$. It is first split up in the typical reusereduce-recycle subgoals, and then decomposed into more fine-grained subgoals.

To design a sustainable activity or system, stakeholders must include this predefined taxonomy as an additional goal and indicate the (positive or negative) contribution of each alternative to the the sustainability softgoals. Often, alternatives that benefit sustainability have a negative impact

2. In this first version, the taxonomy has been driven by the needs of the case study and thus it is not still generic enough. on other softgoals (such as cost or user-experience). See an example (taken from the case study) in Fig. 3. Finally, stakeholders analyze the goal model and reach a consensus on the alternative offering the best trade-off. Existing quantitate and/or qualitative analysis techniques for goalmodels can assist in the process. As an example, the marks in Fig. 3 show that if we opt for organizing an online PC meeting we reduce the pollution (no travel), which is good for sustainability but we endanger the quality of the program.

\section{Case Study: ICSE Conference Organization}

This year's ICSE conference is trying to "go green" by cutting down its ecofootprint. We have been able to apply our method to model the ICSE conference organization process as a way to help in this challenge. In particular, we have developed a set of $i^{*}$ models for the conference. We have depicted the tasks and goals of the conference chairs (and other relevant actors as the attendees) and their effect in our sustainability hierarchy, using negative and postive contribution links. The models are quite large, containing between 36 and 333 elements each.

As sources for our analysis we have used: (1) guidelines for organizing green conferences ${ }^{3}$ (2) our own experience as attendees/organizers of workshops and conferences and, specially, (3) informal communications between and with ICSE chairs.

As an example, Fig. 2 shows the subset of the model corresponding to the general chair. Sustainability appears as one of the softgoals for this chair (part of the higher-level softgoal of having a successful conference), delegated to the (new as of this conference edition) sustainability chair. This delegation implies that the sustainability chair is responsible for developing policies and activities that help satisfy this softgoal. However, the goals of all other chairs have an impact on the achievement of the sustainability softgoal as well, e.g. Fig. 3 shows how a decision regarding the meeting of the PC influences sustainability. Therefore, the selection of the best alternative for each conference aspect must be reached by considering the goals and trade-offs of all actors in the model.

The next step in this project is to validate the models with the conference chairs. Initial feedback has been positive, pointing out that the models are a useful way to present the rationale behind sustainability-related decisions. Our early findings also suggest that goal modeling is indeed useful for the understanding of the domain, for internal communication purposes and for educating people about the effect of their own decisions. We believe that goal models also help to show deficiencies of existing alternatives and foster the search for additional ones which involve better trade-offs.

3. There are many documents and reports available, see www. greeninggovernment.gc.ca/f5b1c0bc-741c-4493-b4b7-b0d56bbe6566/ green_meeting_guide_07.pdf as an example 


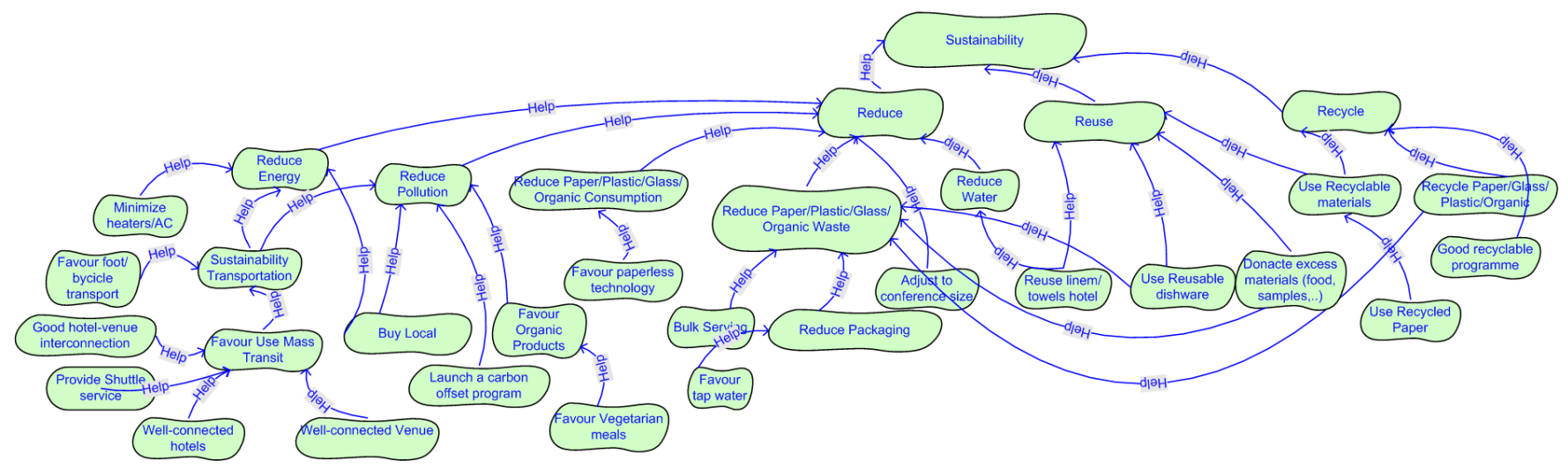

Figure 1. Section of the taxonomy of sustainability-related softgoals (cloud-shaped elements)

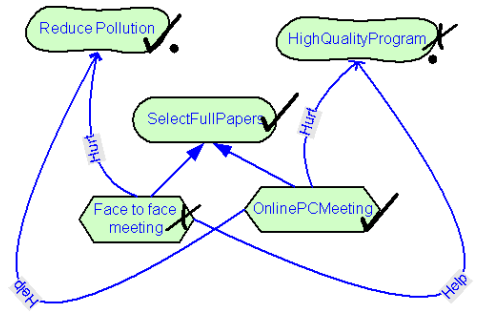

Figure 3. Analysis of alternative tasks (hexagons) that fulfill the select papers goal (oval shape)

\section{Research Challenges}

The goal of this section is to summarize some of the research challenges that need to be addressed to successfully apply our method. Some challenges are related to the sustainability domain in particular but most are relevant to any application of GORE techniques. The list of challenges is the following:

- Lack of standard definitions for sustainability concepts. No general agreements on what policies help sustainability or how to precisely compute the effect of different alternatives (e.g. is it better to publicite sponsors's ads using big screens on the conference site - less paper but more electricity consumption - or by distributing flyers among the attendants- more paper waste but less electricity?).

- Finding the right level of detail for the sustainability taxonomy. The degree of decomposition needed to define and evaluate the effect of the alternatives must be further explored.

- Limitations of sustainability analysis. It is difficult to incorporate quantitative data due to the lack of standard measures for sustainability. On the other hand, qualitative comparisons, althogugh useful, have limitations when deciding between many different alternatives.

- Consistency problems due to the loose semantics of goal models and the different modeling styles. This makes the merge of goal models developed by different designers difficult.
- Scalability problems. Even for small organizations, goal models tend to become huge since they explicitly represent (all) different alternatives. An automatic tool support that allows defining views on the models is clearly needed.

- Validation of the goal models. Once a goal model has been defined, it needs to be reviewed by the stakeholders to be sure that we have correctly collected all requirements. The geographic distribution of the stakeholders and the subjectivity of many decisions (requiring argumentation) makes this process difficult.

\section{Conclusions and Future Work}

We have presented a first attempt to treat sustainability as a first-class element in the early phases of the system/business enginering. Our sustainability taxonomy and the use of GORE techniques allow stakeholders to take into account environmental concerns when making decisions that may have a potential sustainability impact. We believe this represents a first step in the contribution of the software engineering field to the challenge of sustainable development of our society.

As a proof-of-concept we have applied our approach to support the ICSE 2009 greening effort. As a result, our models have revealed several options to reduce the environmental impact of the conference (and their respective trade-offs). Some of the solutions can be easily implemented while others require a stronger community commitment. Therefore, we hope our results serve at least to stimulate discussion among the ICSE community towards a longer term goal of increasing and fostering commitment on sustainability in the following ICSE conferences.

We plan to continue this research line by addressing the challenges outlined before. Moreover we plan to generalize our sustainability taxonomy to cover other business domains and to find analysis approaches that help in the selection of the best alternative under the presence of conflictive requirements. Regarding the validation of goal models, we 


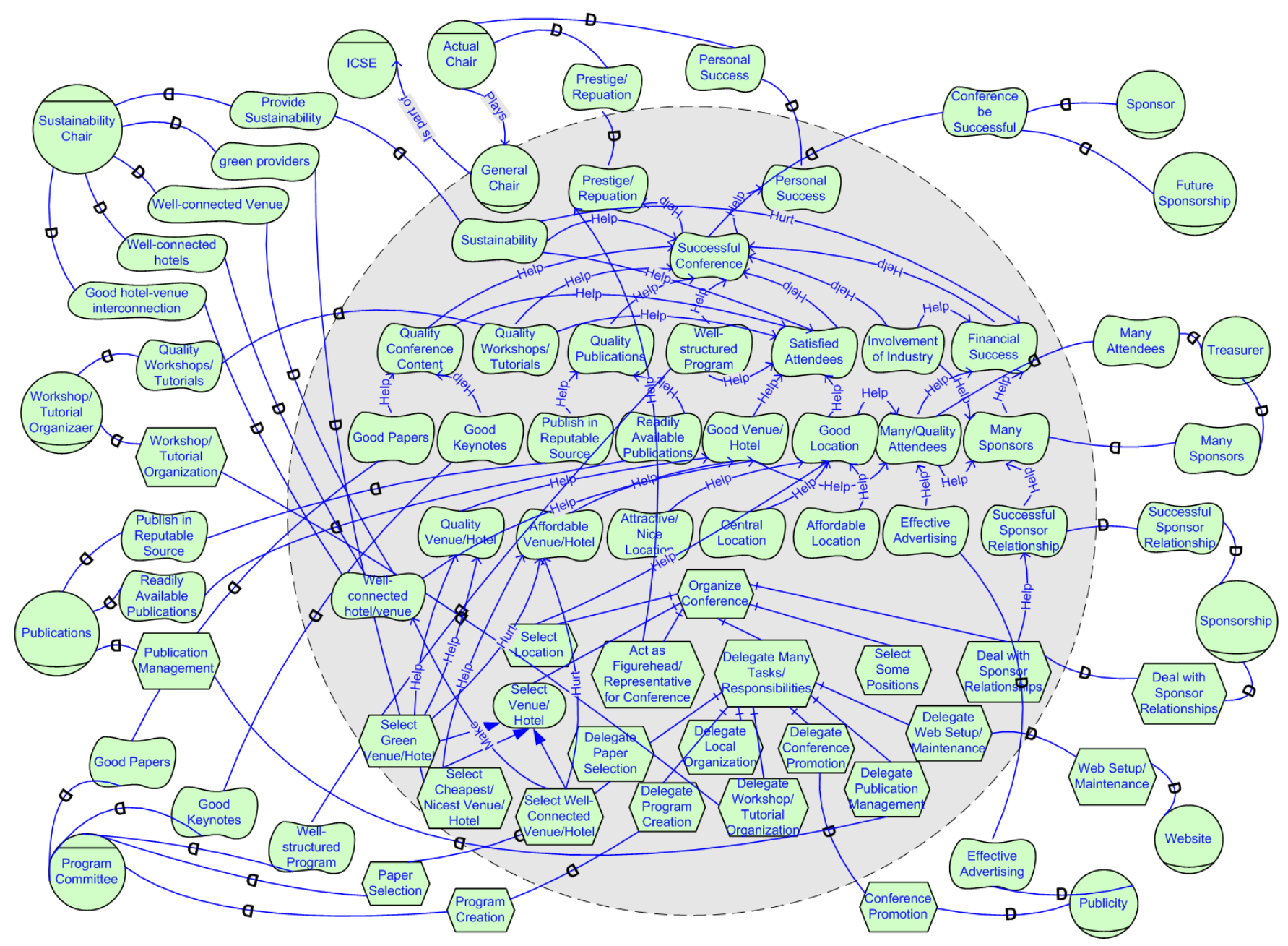

Figure 2. Section of the $i^{\star}$ model for the General Chair actor

plan to test whether an informal validation approach based on the use of wikis/blogs to discuss and comment the models can be useful. Finally, we would like to contact sustainability consultancy companies to apply our approach on industrial case studies.

\section{Acknowledgment}

The authors would like to thank the ICSE 2009 organizers for their help during our work.

\section{References}

[1] H. Sverdrup and M. G. E. Svensson, "Defining the concept of sustainability - a matter of systems thinking and applied systems analysis," in Systems Approaches and Their Application, 2004, pp. 143-164.

[2] U. Nations, World Commission on Environment and Development: Our Common Future. Oxford Univ. Press, 1987.

[3] M. Wackernagel and J. D. Yount, "Footprints for sustainability: the next steps," Environment, Development and Sustainability, vol. 2, no. 1, pp. 21-42, 2000.
[4] E. S. K. Yu, "Towards modeling and reasoning support for early-phase requirements engineering," in 3rd IEEE Int. Symp. on Requirements Engineering, 1997, pp. 226-235.

[5] P. deVoil, W. Rossing, and G. Hammera, "Sustainability tradeoffs in cropping systems using evolutionary algorithms," Environment, Development and Sustainability, vol. 21, no. 9, pp. 1368-1374, 2006.

[6] A. Kurkovsky, "Simulation in the analysis of sustainable development: Goals, planning, and evaluation," in Proc. of the 2004 Int. Conf. on Modeling, Simulation, and Optimization. Acta Press, 2004.

[7] A. Tolon-Becerra and F. Bienvenido, "Conceptual modeling in a meta-model of sustainability indicators," in 12th Int. Conf. on Knowledge-Based Intelligent Information and Engineering Systems, ser. LNCS, vol. 5179. Springer, 2008, pp. 716-723.

[8] G. Regev and A. Wegmann, "Where do goals come from: the underlying principles of goal-oriented requirements engineering," in 13th IEEE Int. Conf. on Requirements Engineering, 2005, pp. 353-362. 